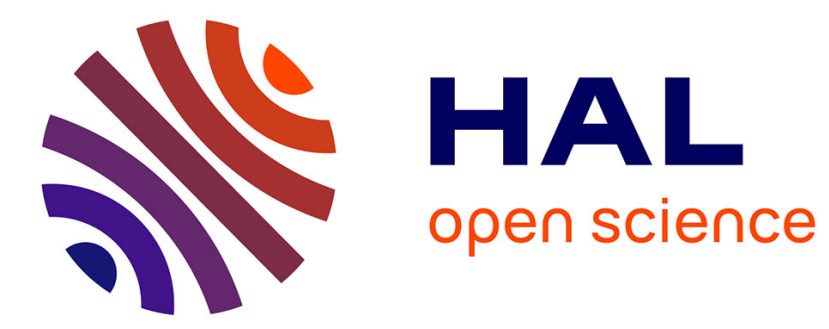

\title{
A NOTE ON THE ASYMPTOTIC VARIANCE OF DRIFT ACCELERATED DIFFUSIONS
}

\author{
Brice Franke, Chii-Ruey Hwang, Hui-Ming Pai, Adel Ouled Said
}

\section{To cite this version:}

Brice Franke, Chii-Ruey Hwang, Hui-Ming Pai, Adel Ouled Said. A NOTE ON THE ASYMPTOTIC VARIANCE OF DRIFT ACCELERATED DIFFUSIONS. 2019. hal-02266338

\section{HAL Id: hal-02266338 \\ https://hal.science/hal-02266338}

Preprint submitted on 14 Aug 2019

HAL is a multi-disciplinary open access archive for the deposit and dissemination of scientific research documents, whether they are published or not. The documents may come from teaching and research institutions in France or abroad, or from public or private research centers.
L'archive ouverte pluridisciplinaire HAL, est destinée au dépôt et à la diffusion de documents scientifiques de niveau recherche, publiés ou non, émanant des établissements d'enseignement et de recherche français ou étrangers, des laboratoires publics ou privés. 


\title{
A NOTE ON THE ASYMPTOTIC VARIANCE OF DRIFT ACCELERATED DIFFUSIONS
}

\author{
B. FRANKE, C.-R. HWANG, A. OULED SAID, AND H.-M. PAI
}

\begin{abstract}
We prove that the asymptotic variance of a drift accelerated diffusion converges to zero uniformly if and only if there are no $H^{1}$-elements in the kernel of the drift generating operator. Our proof is based on spectral analysis in the first order Sobolev space of mean zero functions.
\end{abstract}

\section{INTRODUCTION}

The asymptotic variance is a natural indicator of the efficiency for a Markov Chain Monte Carlo algorithm. The main objective of this paper is to study the behavior of the asymptotic variance for equilibrium preserving perturbations of some reversible diffusion. The analysis is related to the study of antisymmetric perturbations of selfadjoint infinitesimal generators. In this context Hwang, Normand and Wu (2015) obtained some expression for the limit of the asymptotic variance when the amplitude of the drift grows to infinity (see [7]). It was proved by Constantin et al. (2008) in a similar context that the operator norm of the associated semigroups converge to zero if and only if the generator of the drift has no $\mathcal{H}^{1}$ eigenfunctions (see [3]). We will prove an anologue of this result for the case of asymptotic variance. The main objective of the paper is to characterize the drift vector fields having the property that the asymptotic variance goes to zero as the drift increases. It will turn out that asymptotic variance goes to zero uniformly on the unit ball in $\mathcal{L}^{2}$ if and only if there are no $\mathcal{H}^{1}$ eigenfunctions associated to the zero eigenvalue of the drift generating operator. We will study this questions for bounded drift vector fields on compact manifolds and for unbounded drift under some linear growth condition on $\mathbb{R}^{d}$.

\section{Mathematical Setting}

Let $U: \mathbb{R}^{d} \rightarrow \mathbb{R}$ be a given energy function and $\pi$ the probability measure on $\mathbb{R}^{d}$ with density proportional to $e^{-U(x)}$. Let $\mathcal{L}^{2}\left(\mathbb{R}^{2}, \pi\right)$ be the space of complex valued functions which are square-integrable with respect to $\pi$. This space is a Hilbert space endowed with the inner product

$$
<f, g>=\int_{\mathbb{R}^{d}} \bar{f} g d \pi
$$

Date: August 13, 2019.

2010 Mathematics Subject Classification. Primary 60J60; Secondary 60J35, 47D07, 34K08.

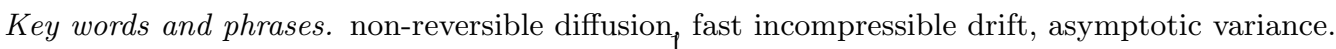


We will consider the space of mean zero functions

$$
\mathcal{H}=\left\{f \in \mathcal{L}^{2}\left(\mathbb{R}^{d}, \pi\right): \int_{\mathbb{R}^{d}} f d \pi=0\right\} .
$$

If one is interested in sampling from $\pi$, then a commonly used process is the time reversible Langevin diffusion having $\pi$ as equilibrium state, which is defined through the following SDE

$$
d X_{t}=-\nabla U\left(X_{t}\right) d t+\sqrt{2} d B_{t},
$$

where $B_{t}$ is a standard Brownian motion. Let $b$ be a weighted divergence free vector field on $\mathbb{R}^{d}$; i.e.: $\operatorname{div}\left(b e^{-U}\right)=0$. Perturbing the reversible diffusion (1) by adding a drift term generated by $b$ results in a SDE of the following form

$$
d X_{t}^{b}=-\nabla U\left(X_{t}^{b}\right) d t+\sqrt{2} d B_{t}+b\left(X_{t}^{b}\right) d t .
$$

It was pointed by Hwang, Hwang-Ma and Sheu (1993), (2005) that a perturbation usually leads to a faster convergence to equilibrium (see [9] and [10]).

We denote by $\mathcal{C}_{c}^{k}\left(\mathbb{R}^{d}\right)$ the space of k-times differentiable functions with compact support. For $f \in \mathcal{C}_{c}^{2}\left(\mathbb{R}^{d}\right)$ the generator of the diffusion defined in (1) has the form

$$
L f=\Delta f-\nabla U \cdot \nabla f .
$$

The operator $L$ is selfadjoint in $\mathcal{L}^{2}\left(\mathbb{R}^{d}, \pi\right)$ and we suppose that its spectrum is discrete with eigenvalues $0=\lambda_{0}>-\lambda_{1} \geqslant-\lambda_{2} \geqslant \ldots$ with $\lambda_{n} \rightarrow \infty$ and associated eigenfunctions $\varphi_{k}, k \in \mathbb{N}$; i.e.: $L \varphi_{k}=\lambda_{k} \varphi_{k}$ for all $k \in \mathbb{N}$. Any function $f \in \mathcal{L}^{2}\left(\mathbb{R}^{d}, \pi\right)$ can be represented in the orthonormal basis $\varphi_{k} ; k \in \mathbb{N}$ as $f=\sum_{k \in \mathbb{N}} \alpha_{k} \varphi_{k}$.

Note that for all $m \in \mathbb{N}$ the spaces

$$
\mathcal{H}^{m}:=\left\{f=\sum_{i=1}^{\infty} \alpha_{i} \varphi_{i} \in \mathcal{H} ; \sum_{i=1}^{\infty}\left|\alpha_{i}\right|^{2} \lambda_{i}^{m}<\infty\right\}
$$

are Hilbert spaces with inner products

$$
\langle f, g\rangle_{m}=\sum_{i=1}^{\infty} \overline{\alpha_{i}} \beta_{i} \lambda_{i}^{m}, \text { where } f=\sum_{i=1}^{\infty} \alpha_{i} \varphi_{i} \text { and } g=\sum_{i=1}^{\infty} \beta_{i} \varphi_{i}
$$

and corresponding norms

$$
\|f\|_{m}^{2}=\sum_{i=1}^{\infty}\left|\alpha_{i}\right|^{2} \lambda_{i}^{m}
$$

Remark 1 : Note that we have the identity

$$
\|f\|_{1}^{2}=\int_{\mathbb{R}^{d}}|\nabla f|^{2} e^{-U} d x .
$$

The fact that this is a norm on $\mathcal{H}^{1}$ follows from the equivalence of $\|f\|_{1}=0$ and $f=0$.

The generator of the modified equation (2) for $f \in \mathcal{C}_{c}^{2}\left(\mathbb{R}^{d}\right)$ has the form

$$
L_{b} f=L f+b \cdot \nabla f \text {. }
$$

Note that the operators $L$ and $L_{b}$ can be generalized to Riemannian manifolds, where they will define manifold valued diffusions $X_{t} ; t \geqslant 0$ resp. $X_{t}^{b} ; t \geqslant 0$. 
Suppose we have for $f \in \mathcal{H}$ that there is a solution $g \in \mathcal{H}^{2}$ to the Poisson equation

$$
L_{b} g=-f
$$

Then the following central limit theorem was proved in Kipnis and Varadhan (1986) and also in Bhattacharya (1982) (see [11] and [1]) :

$$
t^{-\frac{1}{2}}\left(\frac{1}{t} \int_{0}^{t} f\left(X_{s}^{b}\right) d s-\int_{\mathbb{R}^{d}} f d \pi\right) \stackrel{d}{\longrightarrow} N\left(0, \sigma_{b}^{2}(f)\right) \quad \text { as } \quad t \rightarrow 0,
$$

where the asymptotic variance is given by the following expression :

$$
\sigma_{b}^{2}(f)=2\langle f, g\rangle
$$

One can ask the question, how this asymptotic variance behaves as the drift is accelerated to infinity by multiplying the vector field $b$ by a large constant $c$. It was proved in Hwang, Normand and Wu (2015) (see [7]) that

$$
\lim _{|c| \rightarrow \infty} \sigma_{c b}^{2}(f)=2\left\|P_{0} L^{-1 / 2} f\right\|^{2},
$$

where $P_{0}$ is the orthogonal projection in $\mathcal{H}$ on $\mathcal{N}_{0}=\operatorname{ker}\left(L^{-1 / 2} b \cdot \nabla L^{-1 / 2}\right)$. Constantin, Kiselev, Ryzhik and Zlatos (2008) prove that the operator norm of the semi-groups $T_{t}^{(c)}, t \geqslant 0$ generated on $\mathcal{H}$ by the operators $L_{c b}$ converge to zero if and only if the operator $b \cdot \nabla$ has no eigenfunctions in $\mathcal{H}^{1}$ (see [3]). As a consequence of this the spectral gap

$$
\rho_{c b}=-\sup \left\{\operatorname{Re}(z) ; z \in \sigma\left(L_{c b}\right) \backslash\{0\}\right\}
$$

goes to infinity as $|c| \rightarrow \infty$ whenever $b \cdot \nabla$ has no eigenfunctions in $\mathcal{H}^{1}$. It was proved by Franke, Hwang, Pai and Sheu in (2010) (see [6]) that

$$
\lim _{|c| \rightarrow \infty} \rho_{c b}=\inf _{\mu \in \mathbb{R}} \inf \left\{\int_{M}|\nabla \phi|^{2} d \pi ;\|\phi\|=1, \phi \in \mathcal{H}_{\mu}^{1}\right\}
$$

where

$$
\mathcal{H}_{\mu}^{1}=\left\{\phi \in \mathcal{H}^{1}: b \cdot \nabla \phi \stackrel{w}{=} i \mu \phi\right\} .
$$

In this paper we will prove some analogue to the statement of Constantin et al. (2008), for the behavior of the asymptotic variance as $|c| \rightarrow \infty$. It will turn out that $\sup _{\|f\| \leqslant 1} \sigma_{c b}^{2}(f)$ goes to zero if and only if the operator $b \cdot \nabla$ has no $\mathcal{H}^{1}$ eigenfunctions to the eigenvalue zero. This is equivalent to say that there are no $\mathcal{H}^{1}$ functions in the kernel of the operator $b \cdot \nabla$. This shows that asymptotic variance going to zero is a more general phenomenon than spectral gap growing to infinity.

Remark 2: One could think, that since any eigenfunction $\phi \in \mathcal{H}^{1}$ to the eigenvalue $i \mu$ of $b \cdot \nabla$ yields an element from the zero eigenspace by taking absolute value, the condition from Constantin et al. (2008) might be equivalent to ours. However, taking its absolute value might lead to a function which might not be mean zero, and thus is not anymore in $\mathcal{H}^{1}$. 


\section{MAIN RESUlts}

2.1. The case of bounded drift vector fields. In this section, let $M$ be a smooth $d$-dimensional compact connected Riemannian manifold without boundary and let $\Delta$ be the Laplace Beltrami operator on $M$. Again let $U: M \rightarrow \mathbb{R}$ be a $\mathcal{C}^{2}$ function defining a probability density $e^{-U}$ on $M$. Further we suppose that the differential operator

$$
L f=\Delta f-\nabla U \cdot \nabla f
$$

has discrete spectrum and a spectral gap in $\mathcal{H}$. Let $b$ be a $\mathcal{C}^{1}$ vector field satisfying $\operatorname{div}\left(b e^{-U}\right)=0$.

We consider the diffusion generated on $M$ by a differential operator of the form

$$
L_{c b} f=L f+c b \cdot \nabla f \text { for } f \in \mathcal{C}_{c}^{2}(M) .
$$

The following theorem gives a representation for the limit of the asymptotic variance which was published in Duncan et al. (2016) and which is different from the one given in Hwang et al. (2015) (see [5] and [7]). Its proof is based on a method from Bhattacharya, Gupta and Walker (1989) (see [2]). We will repeat it here, since it will serv as a starting point for our investigation. Also note that a similar result was proved in the master thesis of Ouled Said (2016) (see [12]). The following theorem holds :

Theorem 2.1. For any $f \in \mathcal{H}, \sigma_{c b}^{2}(f)$ satisfies

$$
\lim _{|c| \rightarrow \infty} \sigma_{c b}^{2}(f)=2\left\|P L^{-1} f\right\|_{1}^{2},
$$

where $P$ is the orthogonal projection in $\mathcal{H}^{1}$ to $\mathcal{N}_{0}=\operatorname{ker}(b \cdot \nabla) \cap \mathcal{H}^{1}$.

Proof. The following operator was introduced by Bhattachary, Gupta and Walker (see [2]) to study asymptotic diffusion coefficients for homogenized diffusions with large drift $A=L^{-1} b \cdot \nabla: \mathcal{H}^{1} \rightarrow \mathcal{H}^{1}$. They observed that when $b$ is $\mathcal{C}^{1}$ and $\operatorname{satisfies} \operatorname{div}\left(b e^{-U}\right)=0$ then the operator $A$ is antisymmetric and compact. This can be seen as follows :

$\left\langle L^{-1} b \cdot \nabla f, g\right\rangle_{1}=-\langle b \cdot \nabla f, g\rangle=\langle f, b \cdot \nabla g\rangle=-\left\langle f, L^{-1} b \cdot \nabla g\right\rangle_{1}$ for all $f, g \in \mathcal{H}^{1}$.

It is clear that $A$ is compact, because we work on a compact manifold which then implies the compactness of the embedding $\mathcal{H}^{2} \subset \mathcal{H}^{1}$. It follows from those observations that there exist eigenfunctions $\left\{\psi_{k}\right\}_{k \geqslant 1}$ and corresponding eingenvaues $i \mu_{k}$, satisfying $A \psi_{k}=i \mu_{k} \psi_{k}$. Further we have the following properties :

(i) Each $\mu_{k}$ is real and $\lim _{k \rightarrow \infty} \mu_{k}=0$.

(ii) The family $\left\{\psi_{k}\right\}_{k \geqslant 1}$ is a complete orthonormal set in $\mathcal{H}^{1} \cap \mathcal{N}_{0}^{\perp}$, where $\mathcal{N}_{0}^{\perp}$ denotes the orthogonal complement in the Hilbert space $\mathcal{H}^{1}$ of the kernel

$$
\mathcal{N}_{0}:=\left\{h \in \mathcal{H}^{1} ; A h=0\right\} .
$$

(iii) Each $h \in \mathcal{H}^{1}$ can be represented as

$$
h=P h+\sum_{k=1}^{\infty} \alpha_{k} \psi_{k},
$$


where for $k=1,2, \ldots, \alpha_{k}=\left\langle h, \psi_{k}\right\rangle_{1}$ and where $P$ is the orthogonal projection onto $\mathcal{N}_{0}$ in the Hilbert space $H^{1}$. Note that

$$
\|h\|_{1}^{2}=\|P h\|_{1}^{2}+\sum_{k=1}^{\infty}\left|\alpha_{k}\right|^{2} .
$$

It is known that for all $f \in \mathcal{H}$ and all $c \in \mathbb{R}$ there is a solution $g \in \mathcal{H}^{1}$ to the Poisson equation

$$
L_{c b} g=(L+c b \cdot \nabla) g=-f .
$$

Since by elliptic regularity $g \in H^{2}$, we this can be reorganized as follows

$$
g+c A g=g+c L^{-1} b \cdot \nabla g=-L^{-1} f .
$$

We have the representations

$$
g=\sum_{k=1}^{\infty} \beta_{k} \psi_{k}+P g \quad \text { and } \quad L^{-1} f=P L^{-1} f+\sum_{k=1}^{\infty} \alpha_{k} \psi_{k}
$$

with $\alpha_{k}=\left\langle L^{-1} f, \psi_{k}\right\rangle_{1}=\left\langle f, \psi_{k}\right\rangle$ for all $k \in \mathbb{N}$.

Replacing $g$ and $L^{-1} f$ by their respective series representation in equation (3), we obtain :

$$
P g+\sum_{k=1}^{\infty}\left(\beta_{k}+i c \mu_{k} \beta_{k}\right) \psi_{k}=-P L^{-1} f-\sum_{k=1}^{\infty} \alpha_{k} \psi_{k} .
$$

By identification, we obtain

$$
P g=-P L^{-1} f \quad \text { and } \quad \beta_{k}=-\frac{\alpha_{k}}{1+i c \mu_{k}} .
$$

We see that as $c \rightarrow \infty$ we have $\beta_{k} \rightarrow 0$ and it follows that $L_{c b}^{-1} f \rightarrow P L^{-1} f$ in $\mathcal{H}^{1}$ as $c \rightarrow \infty$. Now,

$$
\begin{aligned}
\lim _{|c| \rightarrow \infty} \sigma_{c b}^{2}(f) & =-\lim _{|c| \rightarrow \infty} 2\left\langle L_{c b}^{-1} f, f\right\rangle=-2\left\langle P L^{-1} f, f\right\rangle=2\left\langle L^{-1} P L^{-1} f, f\right\rangle_{1} \\
& =2\left\langle P L^{-1} f, L^{-1} f\right\rangle_{1}=2\left\langle P L^{-1} f, P L^{-1} f\right\rangle_{1}=2\left\|P L^{-1} f\right\|_{1}^{2} .
\end{aligned}
$$

This finishes the proof.

Corollary 2.2. The following two statements are equivalent:

i) $\lim _{|c| \rightarrow \infty} \sigma_{c b}^{2}(f)=0$ for all $f \in \mathcal{H}$;

ii) $\mathcal{H}^{1} \cap \operatorname{ker}(b \cdot \nabla)=\{0\}$.

Proof. F irst we prove (i) $\Longrightarrow$ (ii). We give a proof by contradiction. Suppose there is a $h \in \mathcal{H}^{1} \cap \operatorname{ker}(b \cdot \nabla)$ with $h \neq 0$. We then have for all $f \in \mathcal{H}$

$$
\left\langle P L^{-1} f, h\right\rangle_{1}=\left\langle L^{-1} f, P h\right\rangle_{1}=\left\langle L^{-1} f, h\right\rangle_{1}=-\langle f, h\rangle .
$$

In particular for the choice $h=f$ this yields

$$
0<\|h\|^{2}=\langle h, h\rangle=-\left\langle P L^{-1} h, h\right\rangle_{1} \leqslant\|h\|_{1}\left\|P L^{-1} h\right\|_{1},
$$

which implies $\left\|P L^{-1} h\right\|_{1}>0$, which by Theorem 2.1 would imply $\lim _{c \rightarrow \infty} \sigma_{c b}^{2}(h)>0$. This contradicts (i).

Now we turn to the implication (ii) $\Longrightarrow$ (i). If one has $\mathcal{H}^{1} \cap \operatorname{ker}(b \cdot \nabla)=\{0\}$, then it 
follows that $\operatorname{ker}\left(L^{-1} b \cdot \nabla\right)=\{0\}$. This implies $P L^{-1} f=0$ for all $f \in \mathcal{H}^{1}$. From the previous theorem then follows that $\lim _{c \rightarrow \infty} \sigma_{c b}^{2}(f)=0$ for all $f \in \mathcal{H}^{1}$.

Now we will introduce some results that will be useful later.

Remark 3 : In the following we will use Dini's Theorem ([4] page136) which states that for a compact metric space $K$, a continuous function $F: K \longrightarrow \mathbb{R}$ and a sequence of continuous functions $F_{n}: K \longrightarrow \mathbb{R}, n \in \mathbb{N}$, satisfying that $F_{n}$ converges pointwise to $F$ on $K$ and $F_{n} \geqslant F_{n+1}$ for and all $n \in \mathbb{N}$, we have that $F_{n}$ converges uniformly to $F$ on $K$.

Proposition 2.3. For $\mathcal{K}=\left\{L^{-1} f ; f \in \mathcal{H},\|f\| \leqslant 1\right\}$ the family of functions

$$
G_{c}: \mathcal{K} \longrightarrow \mathbb{R}: h \longmapsto 2 \sum_{k=1}^{\infty} \frac{1}{1+c^{2} \mu_{k}^{2}}\left|\left\langle h, \phi_{k}\right\rangle_{1}\right|^{2}
$$

converges as $|c| \rightarrow \infty$ uniformly on the compact set $\mathcal{K}$ to the zero function.

Proof. Note that the set $\mathcal{K}$ is compact with respect to the $\mathcal{H}^{1}$ norm since $L^{-1} f \in \mathcal{H}^{2}$ and moreover $\left\|L^{-1} f\right\|_{2} \leqslant 1$ when $\|f\| \leqslant 1$, which says that the set $\mathcal{K}$ s bounded in $H^{2}$ and thus compact in $H^{1}$. For $h \in \mathcal{K}$ there exists some $f \in \mathcal{H}$ with $\|f\| \leqslant 1$ and $h=L^{-1} f$. It follows :

$$
\|h\|_{1}^{2}=\left\|L^{-1} f\right\|_{1}^{2}=-\left\langle f, L^{-1} f\right\rangle \leqslant\|f\|\left\|L^{-1} f\right\| \leqslant \frac{1}{\lambda_{1}} .
$$

Let us first prove that $G_{c}$ is continuous with respect to the $\mathcal{H}^{1}$ norm. One has

$$
\begin{aligned}
\left|G_{c}(h)-G_{c}\left(h_{0}\right)\right| & =2\left|\sum_{k=1}^{\infty} \frac{1}{1+c^{2} \mu_{k}^{2}}\left(\left\langle h, \varphi_{k}\right\rangle_{1}^{2}-\left\langle h_{0}, \varphi_{k}\right\rangle_{1}^{2}\right)\right| \\
& \leqslant 2 \sum_{k=1}^{\infty}\left|\left(\left\langle h, \varphi_{k}\right\rangle_{1}-\left\langle h_{0}, \varphi_{k}\right\rangle_{1}\right)\left(\left\langle h, \varphi_{k}\right\rangle_{1}+\left\langle h_{0}, \varphi_{k}\right\rangle_{1}\right)\right| \\
& \leqslant 2\left(\sum_{k=1}^{\infty}\left|\left\langle h-h_{0}, \varphi_{k}\right\rangle_{1}\right|^{2}\right)^{1 / 2}\left(\sum_{k=1}^{\infty}\left|\left\langle h+h_{0}, \varphi_{k}\right\rangle_{1}\right|^{2}\right)^{1 / 2} \\
& =2\left\|h-h_{0}\right\|_{1}\left\|h+h_{0}\right\|_{1} \\
& \leqslant 2\left\|h-h_{0}\right\|_{1}\left(\|h\|_{1}+\left\|h_{0}\right\|_{1}\right) \\
& \leqslant \frac{4}{\lambda_{1}}\left\|h-h_{0}\right\|_{1} .
\end{aligned}
$$

Furthermore, we have for $c_{0}<c_{1}$ that

$$
G_{c_{1}}(h)=2 \sum_{k=1}^{\infty} \frac{1}{1+c_{1}^{2} \mu_{k}^{2}}\left|\left\langle h, \varphi_{k}\right\rangle_{1}\right|^{2} \leqslant 2 \sum_{k=1}^{\infty} \frac{1}{1+c_{0}^{2} \mu_{k}^{2}}\left|\left\langle h, \varphi_{k}\right\rangle_{1}\right|^{2}=G_{c_{0}}(h) .
$$

It is clear that by dominated convergence theorem $G_{c}(h)$ converges to zero as $c \rightarrow \infty$. The statment of the proposition now follows from Dini's theorem.

We now come to our main result. 
Theorem 2.4. For $\mathcal{B}=\{f \in \mathcal{H} ;\|f\| \leqslant 1\}$ the next two statements are equivalent:

i) $\lim _{|c| \rightarrow \infty} \sup _{f \in \mathcal{B}} \sigma_{c b}^{2}(f)=0$;

ii) $\mathcal{H}^{1} \cap \operatorname{ker}(b \cdot \nabla)=\{0\}$

Proof. First we prove (i) $\Longrightarrow$ (ii). Suppose that there is a $f_{0} \in \mathcal{H}^{1} \cap \operatorname{ker}(b \cdot \nabla)$ with $f_{0} \neq 0$. We can suppose without loss of generality that $\left\|f_{0}\right\|=1$. Note that by Corollary 2.2 , we have

$$
\lim _{|c| \rightarrow \infty} \sup _{f \in \mathcal{B}} \sigma_{c b}^{2}(f) \geqslant \lim _{c \rightarrow \infty} \sigma_{c b}^{2}\left(f_{0}\right)>0 .
$$

Let us now prove that (ii) $\Longrightarrow$ (i). We have for $f \in \mathcal{H}$ and $g=-L_{c b}^{-1} f$

$$
\sigma_{c b}^{2}(f)=-2\left\langle L_{c b}^{-1} f, f\right\rangle=2\left\langle L_{c b}^{-1} f, L^{-1} f\right\rangle_{1}=-2\left\langle g, L^{-1} f\right\rangle_{1} .
$$

We saw in the proof ot Theorem 2.1 that we have the following representations in $\mathcal{H}^{1}$

$$
g=\sum_{k=1}^{\infty} \beta_{k} \psi_{k}+P g \quad \text { and } \quad L^{-1} f=P L^{-1} f+\sum_{k=1}^{\infty} \alpha_{k} \psi_{k} .
$$

It follows that

$$
\begin{aligned}
\sigma_{c b}^{2}(f) & =-2\left\langle P g-\sum_{k=1}^{\infty} \frac{\alpha_{k}}{1+i c \mu_{k}} \psi_{k}, P L^{-1} f+\sum_{k=1}^{\infty} \alpha_{k} \psi_{k}\right\rangle_{1} \\
& =-2\left\langle P g, P L^{-1} f\right\rangle_{1}+2 \sum_{k=1}^{\infty} \frac{\alpha_{k}^{2}}{1+i c \mu_{k}} \\
& =2\left\|P L^{-1} f\right\|_{1}^{2}+2 \sum_{k=1}^{\infty} \frac{1}{1+c^{2} \mu_{k}^{2}}\left|\left\langle L^{-1} f, \psi_{k}\right\rangle_{1}\right|^{2} \\
& =2\left\|P L^{-1} f\right\|_{1}^{2}+2 G_{c}\left(L^{-1} f\right) .
\end{aligned}
$$

Since we supposed that $\mathcal{H}^{1} \cap \operatorname{ker}(b \cdot \nabla)=\{0\}$ it follows that $\left\|P L^{-1} f\right\|_{1}=0$. Therefore, by Proposition 2.3, this implies that

$$
\lim _{|c| \rightarrow \infty} \sup _{f \in \mathcal{B}} \sigma_{c b}^{2}(f)=\lim _{|c| \rightarrow \infty} \sup _{f \in \mathcal{B}} G_{c}\left(L^{-1} f\right)=\lim _{|c| \rightarrow \infty} \sup _{h \in \mathcal{K}} G_{c}(h)=0 .
$$

This finishes the proof.

2.2. The case of unbounded drift on $\mathbb{R}^{d}$. In this section, we consider the diffusions (1) and (2) on $\mathbb{R}^{d}$. Let $U: \mathbb{R}^{d} \rightarrow \mathbb{R}$ be a $\mathcal{C}^{2}$ function such that $e^{-U}$ is a probability density and $b$ be a $\mathcal{C}^{1}$ vector field satisfying $\operatorname{div}\left(b e^{-U}\right)=0$.

We consider the diffusion generated on $\mathbb{R}^{d}$ by a differential operator of the form

$$
L_{c b} f=L f+c b \cdot \nabla f \text { for } f \in \mathcal{C}_{c}^{2}\left(\mathbb{R}^{d}\right),
$$

where $L f=\Delta f-\nabla U \cdot \nabla f$ and where $\Delta$ is the Laplace operator on $\mathbb{R}^{d}$. We make the same assumptions as in [7], which are :

(A1) For all $\varepsilon>0$, there is a $c_{\varepsilon}>0$ such that

$$
|b \cdot \nabla U|+\left|D^{2} U\right| \leqslant \varepsilon|\nabla U|^{2}+c_{\varepsilon},
$$

where $D^{2} U$ denotes the Hessian matrix of $U$.

(A2) There is a constant $k>0$ such that $|b| \leqslant k(|\nabla U|+1)$. 
(A3) As $|x| \rightarrow \infty$ one has $|\nabla U(x)| \rightarrow \infty$.

Now we will intoduce some results that will be useful later.

Lemma 2.5. The set $\mathcal{A}=\left\{f \in \mathcal{C}^{1}\left(\mathbb{R}^{d}\right) \cap \mathcal{H}^{1}: \nabla f \in \mathcal{C}_{c}\left(\mathbb{R}^{d}\right)\right\}$ is dense in $\mathcal{H}^{1}$.

Proof. For $f \in \mathcal{H}^{1}$ and $\epsilon>0$ there is $g \in \mathcal{C}^{\infty}\left(\mathbb{R}^{d}\right) \cap \mathcal{H}^{1}$ such that $\|f-g\|_{1} \leqslant \epsilon$.

Let us introduce a new function $g_{r}:=g \phi_{r}$, where we used the truncation function

$$
\phi_{r}(x):= \begin{cases}1 & \text { if } 0 \leqslant|x|<r ; \\ \frac{1}{2}\left(1+\sin \left(\pi|x|+\pi\left(\frac{1}{2}-r\right)\right)\right. & \text { if } r \leqslant|x|<r+1 \\ 0 & \text { if }|x| \geqslant r+1 .\end{cases}
$$

Note that the function

$$
h_{r}:=g_{r}-\int_{\mathbb{R}^{d}} g_{r} e^{-U} d x
$$

is in the set $\mathcal{A}$ for any choice of $r>0$. Further, we have the inequality

$$
\begin{aligned}
\int_{\mathbb{R}^{d}}\left|\nabla h_{r}-\nabla f\right|^{2} e^{-U} d x \leqslant & \int_{B_{r}(0)}|\nabla g-\nabla f|^{2} e^{-U} d x+4 \int_{B_{r+1}(0) \backslash B_{r}(0)}|\nabla g|^{2} e^{-U} d x \\
& +2 \pi \int_{B_{r+1}(0) \backslash B_{r}(0)}|g|^{2} e^{-U} d x+2 \int_{B_{r+1}(0) \backslash B_{r}(0)}|\nabla f|^{2} e^{-U} d x \\
& +\int_{B_{r+1}^{c}(0)}|\nabla f|^{2} e^{-U} d x .
\end{aligned}
$$

Since $\|f\|_{1},\|g\|$ and $\|g\|_{1}$ are all finite it follows that the right side of the above inequality is bouded by $2 \epsilon$ for $r$ sufficienly large.

It follows from the previous lemma and the assumptions on $b$ that the domain of definition $\operatorname{dom}(B)$ of the operator $B=i A=i L^{-1} b \cdot \nabla$ is dense in $\mathcal{H}^{1}$. The following proposition and its proof is a variation of some result from [7].

Proposition 2.6. The operator $B$ is essentially self-adjoint as an operator on $\mathcal{H}^{1}$.

Proof. Since $B$ is symmetric with respect to the scalar product on $\mathcal{H}^{1}$, we need to check that the range range $(B \pm i)$ of $B \pm i$ is dense in $\mathcal{H}^{1}$ (see corollary to Theorem VIII.3 in [8]). For any $g \in \mathcal{H}^{2}$ we have that $L(-i g)$ provides a well-defined element of $\mathcal{H}$. Further, since $L_{b}$ is onto $\mathcal{H}$ there exists a $h \in \mathcal{H}^{2}$ such that $L_{b} h=L(-i g)$. Now, it follows that

$$
L^{-1} b \cdot \nabla h=L^{-1}\left(L_{b}-L\right) h=L^{-1} L_{b} h-h=L^{-1} L(-i g)-h=-i g-h .
$$

We conclude that

$$
(B+i) h=i\left(L^{-1} b \cdot \nabla h+h\right)=i(-i g-h+h)=g .
$$

This prove that range $(B+i)=\mathcal{H}^{2} \subset \mathcal{H}^{1}$. This shows that the range of $B+i$ is dense in $\mathcal{H}^{1}$. Similarly, we can show that range $(B-i)=\mathcal{H}^{2} \subset \mathcal{H}^{1}$, by considering $h \in \mathcal{H}^{2}$ such that $L_{b} h=L(i g)$ and since $-L^{-1} b \cdot \nabla=L^{-1}\left(L_{-b}-L\right)$.

Let us give a result concerning the limiting behavior of the asymptotic variance as the magnitude of the perturbation goes to infinity. 
Theorem 2.7. Assume $(\boldsymbol{A} 1),(\boldsymbol{A} 2),(\boldsymbol{A} 3)$. For any $f \in \mathcal{H}^{1}$ we have

$$
\lim _{|c| \rightarrow \infty} \sigma_{c b}^{2}(f)=2\left\|P L^{-1} f\right\|_{1}^{2},
$$

where $P$ is the orthogonal projection in $\mathcal{H}^{1}$ to $\mathcal{N}_{0}=\operatorname{ker}(b \cdot \nabla) \cap \mathcal{H}^{1}$.

Proof. Under the conditions (A1),(A2),(A3) we know that for all $f \in \mathcal{H}$ there is a solution $g \in \mathcal{H}^{1}$ to the Poisson equation $L_{c b} g=-f$ (see [7]). Let $\mu_{L^{-1} f}$ be the spectral measure of the function $L^{-1} f$ associated with the essentially self adjoint operator $B$ on $\mathcal{H}^{1}$ (see [8] page 224). The measure $\mu_{L^{-1} f}$ is supported on the spectrum $\sigma(B)$ of the operator $B$. On the space $\mathcal{H}$ the operators $L$ and $L_{c b}$ are invertible and we have the equality

Since we have

$$
L_{c b}^{-1}=\left(I-c L^{-1} b \cdot \nabla\right)^{-1} L^{-1}
$$

$$
\begin{aligned}
\sigma_{c b}^{2}(f)=-2\left\langle L_{c b}^{-1} f, f\right\rangle & =2\left\langle L_{c b}^{-1} f, L^{-1} f\right\rangle_{1}=2\left\langle\left(I-c L^{-1} b \cdot \nabla\right)^{-1} L^{-1} f, L^{-1} f\right\rangle_{1} \\
& =2\left\langle(I+i c B)^{-1} L^{-1} f, L^{-1} f\right\rangle_{1}=2 \int_{\sigma(B)} \frac{1}{1+i c y} \mu_{L^{-1} f}(d y) .
\end{aligned}
$$

Since we have that the asymptotic variance is real valued it follows that

$$
\begin{aligned}
\sigma_{c b}^{2}(f) & =2 \int_{\sigma(B)} \frac{1}{1+c^{2} y^{2}} \mu_{L^{-1} f}(d y) \\
& =2 \mu_{L^{-1} f}(0)+2 \int_{\sigma(B) \backslash\{0\}} \frac{1}{1+c^{2} y^{2}} \mu_{L^{-1} f}(d y) \\
& =2\left\|P L^{-1} f\right\|_{1}^{2}+2 \int_{\sigma(B) \backslash\{0\}} \frac{1}{1+c^{2} y^{2}} \mu_{L^{-1} f}(d y) .
\end{aligned}
$$

The last equality follows since $P$ is the $\mathcal{H}^{1}$ projection on $\operatorname{ker}(b \cdot \nabla) \cap \mathcal{H}^{1}=\operatorname{ker}(B)$, which is the eigenspace to the zero eigenvalue of $B$. By dominated convergence theorem, the integral vanishes in the limit as $|c| \rightarrow \infty$ and it follows that

$$
\lim _{|c| \rightarrow \infty} \sigma_{c b}^{2}(f)=2\left\|P L^{-1} f\right\|_{1}^{2} .
$$

This proves the theorem.

Now let us show that the asymptotic variance goes to zero uniformly on the unit ball in $\mathcal{H}^{1}$ as the drift increases. Note that the assertion of Corollary 2.2 remains valid also in the current setting of unbounded drift.

Proposition 2.8. Suppose $\mathcal{H}^{1} \cap \operatorname{ker}(b \cdot \nabla)=\{0\}$ and let $\mathcal{K}=\left\{L^{-1} f ; f \in \mathcal{H},\|f\| \leqslant 1\right\}$. The family of functions

$$
F_{c}: \mathcal{K} \longrightarrow \mathbb{R}: h \longmapsto 2 \int_{\sigma(B) \backslash\{0\}} \frac{1}{1+c^{2} y^{2}} \mu_{h}(d y)
$$

converges as $c \rightarrow \infty$ uniformly on the compact set $\mathcal{K}$ to the zero function.

Proof. It follows from the condition on the kernel of $b \cdot \nabla$ that $\operatorname{ker}(B)=\{0\}$ in $\mathcal{H}^{1}$. Therfore, the spectral measure $\mu_{L^{-1} f}$ has no atom in zero. It follows that

$$
F_{c}(h)=\int_{\sigma(B) \backslash\{0\}} \frac{1}{1+c^{2} y^{2}} \mu_{h}(d y)=\int_{\sigma(B)} \frac{1}{1+c^{2} y^{2}} \mu_{h}(d y)=\left\langle\left(I+c^{2} B^{2}\right)^{-1} h, h\right\rangle_{1} .
$$


We first prove that $F_{c}$ is continuous with respect to the $\mathcal{H}^{1}$ norm. Note that we already saw in the proof of Theorem 2.1 that for all $h \in \mathcal{K}$ we have $\|h\|_{1}^{2} \leqslant \frac{1}{\lambda_{1}}$. Since the operator $\left(I+c^{2} B^{2}\right)^{-1}$ is symmetric in $\mathcal{H}^{1}$, one then has for all $h, h_{0} \in \mathcal{K}$

$$
\begin{aligned}
& \left|F_{c}(h)-F_{c}\left(h_{0}\right)\right| \\
= & 2\left|\left\langle\left(I+c^{2} B^{2}\right)^{-1} h, h\right\rangle_{1}-\left\langle\left(I+c^{2} B^{2}\right)^{-1} h_{0}, h_{0}\right\rangle_{1}\right| \\
= & 2\left|\left\langle\left(I+c^{2} B^{2}\right)^{-1} h, h-h_{0}\right\rangle_{1}+\left\langle\left(I+c^{2} B^{2}\right)^{-1}\left(h-h_{0}\right), h_{0}\right\rangle_{1}\right| \\
= & 2\left|\left\langle\left(I+c^{2} B^{2}\right)^{-1} h, h-h_{0}\right\rangle_{1}+\left\langle h-h_{0},\left(I+c^{2} B^{2}\right)^{-1} h_{0}\right\rangle_{1}\right| \\
\leqslant & 2\left(\left\|\left(I+c^{2} B^{2}\right)^{-1} h\right\|_{1}+\left\|\left(I+c^{2} B^{2}\right)^{-1} h_{0}\right\|_{1}\right)\left\|h-h_{0}\right\|_{1} \\
= & 2\left(\sqrt{\int_{\sigma(B)} \frac{1}{\left(1+c^{2} y^{2}\right)^{2}} \mu_{h}(d y)}+\sqrt{\int_{\sigma(B)} \frac{1}{\left(1+c^{2} y^{2}\right)^{2}} \mu_{h_{0}}(d y)}\right)\left\|h-h_{0}\right\|_{1} \\
\leqslant & 2\left(\sqrt{\int_{\sigma(B)} \mu_{h}(d y)}+\sqrt{\int_{\sigma(B)} \mu_{h_{0}}(d y)}\right)\left\|h-h_{0}\right\|_{1} \\
\leqslant & 2\left(\|h\|_{1}+\left\|h_{0}\right\|_{1}\right)\left\|h-h_{0}\right\|_{1} \\
\leqslant & \frac{4}{\lambda_{1}}\left\|h-h_{0}\right\|_{1} .
\end{aligned}
$$

This proves the continuity. Moreover, the monotonicity holds, since for $c_{0}<c_{1}$ we have

$$
F_{c_{1}}(h)=2 \int_{\sigma(B)} \frac{1}{1+c_{1}^{2} y^{2}} \mu_{h}(d y) \leqslant 2 \int_{\sigma(B)} \frac{1}{1+c_{0}^{2} y^{2}} \mu_{h}(d y)=F_{c_{0}}(h) .
$$

On the other hand, by dominated convergence theorem $F_{c}(h)$ converges to zero as $|c| \rightarrow \infty$. By Dini's theorem the result follows.

We now come to our main result in this section.

Theorem 2.9. Assume $(\boldsymbol{A 1}),(\boldsymbol{A} 2),(\boldsymbol{A} 3)$. For $\mathcal{B}=\{f \in \mathcal{H} ;\|f\| \leqslant 1\}$, the following two statements are equivalent:

i) $\lim _{|c| \rightarrow \infty f \in \mathcal{B}} \sup _{c b}^{2}(f)=0$.

ii) $\mathcal{H}^{1} \cap \operatorname{ker}(b \cdot \nabla)=\{0\}$.

Proof. The implication (i) $\Longrightarrow$ (ii) follows from the same arguments as in Theorem 2.1. To finish the proof, let us prove that (ii) $\Longrightarrow$ (i). We have by Theorem 2.7 for $f \in \mathcal{H}$ that

$$
\sigma_{c b}^{2}(f)=2\left\|P L^{-1} f\right\|_{1}^{2}+2 \int_{\sigma(B) \backslash\{0\}} \frac{1}{1+c^{2} y^{2}} \mu_{L^{-1} f}(d y)=2\left\|P L^{-1} f\right\|_{1}^{2}+2 F_{c}\left(L^{-1} f\right) .
$$

From (ii) follows that $\left\|P L^{-1} f\right\|_{1}=0$. Therefore, by Proposition 2.8 , this implies that

$$
\lim _{|c| \rightarrow \infty} \sup _{f \in \mathcal{B}} \sigma_{c b}^{2}(f)=\lim _{|c| \rightarrow \infty} \sup _{f \in \mathcal{B}} F_{c}\left(L^{-1} f\right)=\lim _{|c| \rightarrow \infty} \sup _{h \in \mathcal{K}} F_{c}(h)=0 .
$$

This proves the remaining implication. 


\section{ACKNOWLEDGEMENTS}

Brice Franke wants to thank Academia Sinica in Taiwan for its iterated hospitality, which made this research possible. Adel Ouled Said was supported by the french tunisian research project PHC Utique 16G1505.

\section{REFERENCES}

[1] R.N. Bhattacharya : On the functional central limit theorem and the law of the iterated logarithm for Markov processes. Zeitschrift für Wahrscheinlichkeitstheorie und Verwandte Gebiete, 60(2), 185-201, (1982).

[2] R.N. Bhattacharya, V.N. Gupta and H.K Walker : Asymptotics of solute dispersion in periodic porous media. SIAM Journal on Applied Mathematics, 49, 86-98, (1989)

[3] P. Constantin, A. Kiselev, L. Ryzhik and A. Zlatos : Diffusion and mixing in fluid flow. Annals of Mathematics, 168(2), 643-674, (2008).

[4] J. Dieudonné : Eléments d'analyse. Fondements de l'analyse moderne, Tome I, GauthierVillars, Paris, (1981).

[5] A. Duncan, T. Lelievre and G. Pavliotis : Variance reduction using nonreversible langevin samplers. Journal of Statistical Physics, 163, 457-49, (2016).

[6] B. Franke, C.-R. Hwang, H.-M. Pai, and S.-J. Sheu : The behavior of the spectral gap under growing drift. Transactions of the American Mathematical Society, 362(3), 1325-1350, (2010).

[7] C.-R. Hwang, R. Normand . and S-J. Wu : Variance reduction for diffusions. Stochastic Processes and their Applications, 125(9), 3522-3540, (2015).

[8] M. Reed And B. Simon : Methods of Modern Mathematical Physics I : Functional Analysis. New York-London, (1975).

[9] C.-R. Hwang, S.-Y. Hwang-Ma And S.-J. Sheu : Accelerating Gaussian diffusions. Annals of Applied Probabiity, 3(3), 897-913, (1993).

[10] C.-R. Hwang, S.-Y. Hwang-Ma And S.-J. Sheu : Accelerating diffusions. Annals of Applied Probabability, 15(2), 1433-1444, (2005).

[11] C. Kipnis and S.R.S. Varadhan : Central limit theorem for additive functionals of reversible Markov processes and applications to simple exclusions. Communications in Mathematical Physics 104(1), 1-19, (1986).

[12] A. Ouled SAID : Étude de la variance asymptotique pour la diffusion irréversible. Master Thesis. Sfax University, (2016).

Brice Franke, Laboratiore de Mathématiques de Bretagne Atlantique UMR 6205, UfR Sciences et Techniques, Université de Bretagne Occidentale, 6 Avenue Le Gorgeu, CS 93837, 29238 BRest, CEDEX 3, France

E-mail address: brice.franke@univ-brest.fr

Chil-Ruey Hwang,Institute of Mathematics, Academia Sinica, 6F, Astronomy-Mathematics Building, No. 1, Sec. 4, Roosevelt Road, Taipei 10617, Taiwan

E-mail address: crhwang@sinica.edu.tw

Adel Ouled Said, Laboratoire D’Algèbre Géométrie et Théorie Spectrale RL/11/E553, Département de Mathématiques, Faculté des Sciences de Sfax, Université de Sfax, 3000 SFAX, Tunisia

E-mail address: adel.ouledsaid@univ-brest.fr

Hui-Ming Pai, Department of Statistics, National Taipei University, San Shia, 237, TAIWAN

E-mail address: hpai@mail.ntpu.edu.tw 